\title{
Recent Development Applications of Nanotechnology in Cancer: A Literature Review
}

\author{
P. Selvakumari \\ Department of Physics, Periyar Arts College, Cuddalore-1, India \\ *Corresponding Author: manavalanscholar@gmail.com \\ Available online at: www.isroset.org \\ Received: 05/Dec/2018, Accepted: 24/Dec/2018, Online: 31/Dec/2018
}

\begin{abstract}
Oral health could be maintained by application of this technology in prevention, diagnosis and treatment. Oral cancer is a debilitating disease, and numerous research activities are being pursued worldwide to combat this deleterious process. Nanotechnology is very diverse field that has revolutionized the industry and is setting new trends in the management of oral cancer. Hence, we performed a PubMed search on nanotechnology in oral cancer and found 211 articles related to this search. We have reviewed the reported literature to the best of our abilities and summarized the various aspects of nanotechnology, its role in diagnosis - nanodiagnostics and treatment of oral cancer - nanotherapeutics in this article. Nanotechnology for oral cancer could be a use full tool for detection and drug therapy. By Possessing unprecedented potential for early stage detection, treatment of oral cancer, nanoparticles have been demonstrated over the last decade. In this review, we will summarize the current state-of-the-art of nanoparticles in biomedical applications such as detection, diagnosis and targeting over the oral cancer. Gold nanospheres, nanorods, nanoshells, nanocages, and surface enhanced nanoparticles will be discussed in detail regarding their uses in vivo imaging, cancer therapy, and drug delivery. The key feature of nanoparticlebased drug agents to target over ligands, therapeutic drugs, and other functionalities can all be integrated to allow for targeted molecular imaging and molecular therapy of oral cancer. The future of the nanotechnology for oral cancer looks brighter than ever yet many hurdles remain to be conquered. A multifunctional platform based on gold nanoparticles, with multiple receptor targeting, multimodality imaging, and multiple therapeutic entities, holds the promise for a "magic gold bullet" against oral cancer.
\end{abstract}

Keywords - Diagnosis, nanotechnology, oral cancer, therapy

\section{INTRODUCTION}

Cancer is a critical public health problem worldwide that has brought great burden to society. In 2016, an estimated $1,685,210$ new cases and 595,690 cancer deaths occurred in the United States alone [1]. Oral cancer is the sixth most common cancer globally and has a 5-year survival rate of around 50\% [2]. According to US cancer statistics, approximately 31,910 new cases of oral cancer and 6490 oral cancer deaths occurred in 2016 [3]. Oral cancer is an aggressive cancer that mainly affects oral epithelial cells, may develop metastasis, and even results in death [4]. The major type of malignancy is oral squamous cell carcinomas (OSCC), which accounts for more than $90 \%$ of all oral cancers [5]. These tumors may invade the mucosa of the tongue, buccal, floor of mouth, alveolar and the hard palate, and the tongue is reported to be the most common subsite, with poor prognosis $[1,6]$. Oral carcinogenesis is often due to long-term exposure to various potential risk factors, which may lead to accumulation of multiple genetic mutations [4].
Several major risk factors for oral cancer, including smoking, alcohol consumption, and human papillomavirus infection, with smoking acting as the leading cause of cancer death $[3,7]$. Besides, habitual use of the areca nut is another risk factor that closely associated with oral cancer, especially in Indian subcontinent [8].

The formation of oral cancer is a multifactorial and multistep process [6]. Oral leukoplakia, oral erythroplakia, oral lichen planus, oral submucous fibrosis, actinic keratosis, and discoid lupus erythematosus are common oral potentially malignant disorders (OPMD) that are known to have the potential for malignant transformation [8,9]. Thus, early detection of OPMD and oral cancer is critical for the prognosis of diseases [5]. To date, scalpel biopsy and histopathological examinations are still the standard diagnostic procedures applied to ascertain the oral potentially malignant and malignant lesions $[17,18]$. However, the biopsy procedure is often invasive, which may cause patients anxiety and discomfort [10]. The selection of resection 
margins depends largely on the histopathological assessments, and the results can be affected by the quality of the specimens and pathologists' subjective judgments $[11,12]$. In addition, the assessments are unable to detect small numbers of genetically abnormal cells at the margins, thus leaving the risk of recurrence $[13,14]$.

According to the US National Nanotechnology Initiative, nanotechnology refers to the manipulation of matter with the length scale of $1-100 \mathrm{~nm}$ in at least one dimension $[37,38]$. In the past few decades, nanotechnologies have been applied in various fields, especially in the medical field [39]. One of the most hotly researched subfield of nanotechnology is nanomedicine, which increases the possibility of specific targeted cancer therapy [40]. Moreover, nanotechnology is also a useful tool for cancer detection, and monitoring the disease as it metastasizes [41, 42, 43, 44]. To date, nanotechnology has been applied in the detection and diagnosis of various cancers, such as cervical cancer, lung cancer, breast cancer, gastric cancer, nasopharyngeal cancer, and oral cancer $[45,46,47,48,49,50,51,52]$. As far as we know, the application of nano-based detection methods for oral cancer has not been systematically reviewed. In this review, we highlighted the various nanotechnologies that have been developed for oral cancer detection and diagnosis. The application of nanotechnology for in vitro and in vivo bioimaging of oral cancer was shown in Fig. 1.

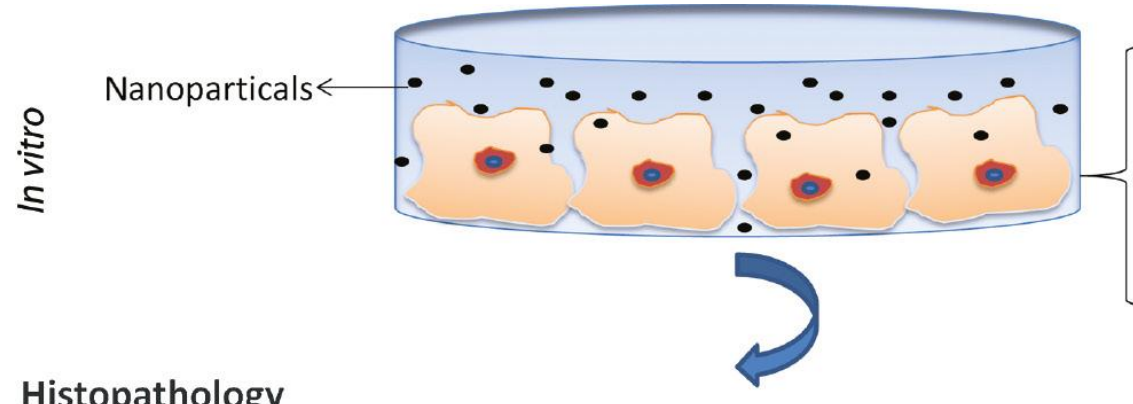

Histopathology

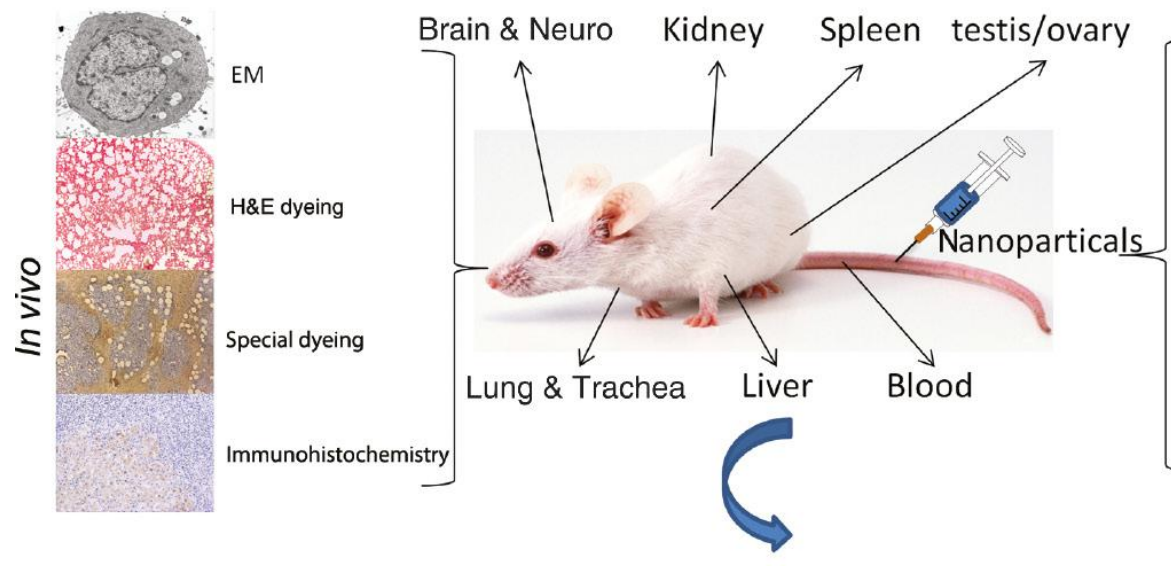

Cell proliferation

Apoptosis and necrosis DNA synthesis and damage

Altered gene expression Immunogenicity Oxidative stress

\section{Further applications}

Figure 1: Application of nanotechnology for in vitro and in vivo bioimaging of oral cancer

\section{CONVENTIONAL DETECTION AND TREATMENT OF CANCER}

Detection of cancer is completed by remarking the physical changes in the body part using X-rays or CT scans and is later justified by biopsy through cell culture. But this method is not very responsive, and the detection is possible after considerable growth of the cancerous cells (Chandrasekhar P
\& M, 2013). The treatment options are chemotherapy (Gorka Orive, 2005) surgery (Jabir et al., 2012), irradiation therapy (C. Moorthi, 2011), or a combination approach (Jabir et al., 2012), (Birendra Kumar, 2009). Surgery means removal of cancerous part. When the cancerous cells (CC) are burnt by specific frequency radiation, it is called radiation therapy and when those (CC) are killed by drugs toxic to cells, is termed as chemotherapy (Jabir et al., 2012). Conventional treatment method has several limitations like, systemic toxicity 
(chemotherapy) (Vergaro et al., 2011), the treatment may not possible for all types of cancer cases (surgery) (Jabir et al., 2012), radiation therapy may cause toxicity (Gunasekera, Pankhurst, \& Douek, 2009), gross approach and may unsuccessful in case of advanced stage of the cancer (chemotherapy) (Vergaro et al., 2011). Until now, chemotherapy is the widely used method to treat localized and metastasized cancer. But because of its several limitations (limited aqueous solubility, lack of selectivity of anticancer drugs, multidrug resistance etc) it is not very effective (Vergaro et al., 2011), (Jones \& Saba, 2011).

\section{A. CANCER NANOTECHNOLOGY}

Cancer nanotechnology is the medical application of nanotechnology, which is a new interdisciplinary field where prevention, diagnosis and treatment of cancer have done with the help of nanotechnology (Santos, Ponte, Boonme, Silva, \& Souto, 2013),(Misra, Acharya, \& Sahoo, 2010),(Cai, Gao, Hong, \& Sun, 2008). In comparison with the traditional way this new technology, also known as "nanooncology" holds much promise (Meng et al., 2012), (K. K. Jain, 2010) that shows scientist new hope to deal with cancer (Patra, Bhattacharya, Mukhopadhyay, \& Mukherjee, 2010).

\section{B.TYPES OF PHARMACEUTICAL NANOSYSTEMS WITH THEIR APPLICATIONS}

The following are different types of nanosystems with their pharmaceutical applications

\section{A.Dendrimers}

Dendrimers are globular, highly branched, and synthetic polymers containing of an initiator core and multiple layers with active terminal groups. These layers are comprised of repeating units and each of these layer is called a generation. The core of a dendrimer is indicated as generation zero. The specific molecular structureof dendrimers with interior coremakes them suitable to carry various drugs using their multivalent surfaces throughelectrostatic adsorption or covalent conjugation. The advantages of dendrimers are that they are uniform in size to many proteins and biomolecules like insulin, and haemoglobin. Second generation dendrimers have a width similar to that of DNA $(2.4 \mathrm{~nm})$. Through hydrophobic interaction, hydrogen bond, or chemical linkage drugs can be loaded in the cavities in dendrimer cores. Researchers in Michigan developed a polyamidoamine-based G5 dendrimer for targeted drug delivery to tumour.The polyamidoamine-based G5 dendrimer has a diameter of about $5 \mathrm{~nm}$ and more than 100 functional primary amines on the surface. By attaching folate as the targeting molecule and methotrexate as the therapeutic agent, the G5 dendrimer was many folds more effective than methotrexate alone in prohibiting tumourgrowth [9]. Dendrimers used in drug delivery and imaging are usually 10 to $100 \mathrm{~nm}$ in diameter with multiple functional groups on their surface, makes them ideal carriers for targeted drug delivery [10]. However, Dendrimers with poly cationic surface shown great potential in the targeted delivery of anticancer therapeutic agents, which can form multiple interactions with a number of target receptors. The poly cationic surface is also having main disadvantage in therapeutic delivery applications are due to their toxic effect on cell membranes [11].

\section{B. Liposomes}

In 1976 liposomes was first described as lipid vesicles that was applied in drug delivery [11].In 1995 liposomes were the first nanoscale drug delivery devices gets approval for clinical use. Since then, up to today many of developments are takes place in liposomes drug delivery system. Now a days liposomes are available not only for oral but also for topical and transdermal route. Long circulating liposomes, stimuli-responsive liposomes, elastic liposomes, nebulized liposomes are the new advances of liposomal drug delivery system [12]. Liposomes are spherical vesicles composed of amphiphilic phospholipids and cholesterol, which selfassociate into bilayers to encapsulate an aqueous interior. The amphiphilic phospholipid molecules form a closed bilayer sphere in an attempt tostill maintaining contact with the aqueous phase via the hydrophilic head group, while shield their hydrophobic groups from the aqueous environment. Because a liposome can encapsulate an aqueous solution with a lipophilic outer membrane, hydrophilic solutes cannot pass through the lipids. So, liposomes can carry both lipophilic molecules as outer membrane and hydrophilic molecules in the inner aqueous core. Liposomes can be classified into three categories depending upon their size and number of bilayers as: multilamellar vesicles (multi bilayer), large unilamellar vesicles (large size, single bilayer), and small unilamellar vesicles (small size, single bilayer). Liposomes can be divided into five types based upon composition as well as mechanism of intracellular delivery: conventional liposomes, $\mathrm{pH}$-sensitive liposomes, cationic liposomes, immune liposomes, and long-circulating liposomes [10]. The liposomeslipid bilayer fuse with bilayers of the cell membrane delivers its contents to the appropriate area. Liposomes have been intensivelyinvestigated for their use in cancer therapy. The effectiveness of drug delivery systems due to their small size, controlled time release of the drug, modification of drug pharmacokinetics, biological distribution and reduced drug toxicity [3].

\section{C.Quantum dots}

Quantum dots are nanocrystal or small tiny particles a semi conducting material with 2-10 nanometer diameter. Quantum dots are first discovered in 1980. Quantum dots are used for periods ranging from milliseconds to minutes to track individual glycine receptors (GlyRs) and to analyse their 
dynamics in the neuronal membrane of living cells [14]. In recent years, semiconductor quantum dots have attracted the attention of many research groups because of their applications in scientific and technological significance in microelectronics, optoelectronics and cellular imaging [4]. Quantum Dots are semi conducting materials consisting of a semiconductor core coated by a shell to improve optical properties. Their properties originate from their physical size which ranges from 20-200A in diameter [7]. Quantum dots are widely used in biological applications that require fluorescence, including cell biology and immune fluoresence assays, DNA array technology, particularly in the immune staining of proteins, microtubules and nuclear antigens [15]. The most commonly used Quantum dots are cadmium telluride, cadmium selenide, indium, arsenide and indium phosphide. In bio-imaging these particles serve as contrast agents, providing much greater resolution than existing fluorescent dyes. Quantum Dots particles absorbing white light and re-emit it with different bulk band gap energies corresponding to different combinations of particles within nanoseconds [10].

\section{Carbon Nanotubes}

Carbon nanotubes are hexagonal networks of carbon atoms. Carbon nanotubes are $1 \mathrm{~nm}$ in diameter and $1-100 \mathrm{~nm}$ in length. Nanotubes are of two type's single walled nanotubes and multi walled nanotubes. These are small macro molecules have unique size, shape and remarkable physical properties [16]. Carbon nanotubes are carbon cylinders composed of benzene rings. Carbon nanotubeshave been used as diagnostic devices for the discrimination of different proteins from serum samples, biological sensors for detecting protein and DNA, and as carriers to deliver drug, protein or vaccine Single-walled carbon nanotubes have been usedto develop highly specific electronic biomolecule detectors as well as a platform for investigating surface protein and protein binding [14].

\section{E. Polymeric Nanoparticles}

Polymeric nanoparticles having some inherent properties like biocompatibility, biodegradability, non-toxicity and nonimmunogenicity [7]. Polymeric nanoparticles are the combined name for nanospheres and nanocapsules [11]. Polymeric nanoparticles are solid colloidal particle with radius ranging from 0.5 to $500 \mathrm{~nm}$ [17]. Polymeric nanoparticles are developed as effective delivery vehicles because itsability to enhance the efficacy and minimizes the side effects of chemotherapeutic drugs due to their passive tumour-targeting properties. Polymeric nanoparticles having preferentially capacity to accumulate in and around the tumour mass also gives a platform for improved diagnostics of tumour, hereby this property laying the foundation for the new development of multi-functional nanoparticle systems in cancer diagnosis \&therapy [18]. For preparation of polymeric nanoparticles natural macromolecules, such as proteins and polysaccharides, non-polar lipids, metal oxides and silica, and Polymers like poly (alkylcyanocrystalates), poly (mythylidenemalnolate 2.1.2), polyesters, e.g., poly (lactic acid), poly(e-caprolactone), and their copolymers are used [19]. Polymeric nanoparticles can be used for better application by overcoming obstacles in conventional drug delivery \& effective drug delivery and would enhance treatment \& patient compliance [20].

\section{F.Polymeric Micelles}

Now a day's these micelles have emerged as a new promising colloidal carrier for targeted delivery of poor water soluble as well as amphiphilic drugs. Polymeric micelles can enhance solubilisation of hydrophilic compounds in their inner core. These are more stable as compared to surfactant micelles [21]. A polymer micelle is a nanoparticle consisting of two main parts one hydrophilic shell and one hydrophobic core. It can be divided into two main categories: hydrophobically assembled micelles and polyion-complex micelles. The hydrophobically assembled micelles consist of amphiphilic copolymers with a hydrophobic and a hydrophilic block. Balance between those two blocks in an aqueous medium induces spontaneous formation of nano-sized particulates. For most block copolymers, poly (ethylene glycol) is used as a hydrophilic block. Different micelle properties originate from the nature of hydrophobic core-forming materials, which include biodegradable polyesters polymers such as poly (glycolic acid), poly (lactic acid), and poly (e-caprolactone) [22]. These are usually of less than $100 \mathrm{~nm}$ and their hydrophilic surface inhibit uptake by reticuloendothelial system. Micelles formed in solutions as aggregates in which the component molecules are arranged in a spherical structure with hydrophobic core shield from water by a mantle of hydrophilic groups. These are used for systemic delivery of water insoluble drugs [7].

\section{G.Metallic Nanoparticle}

Metallic nanoparticle can be prepared by various methods by using metals like gold etc. A metal nanoparticle shows similar optical properties which is dependent upon shape and size [23]. Researcher made nanoparticle by using various metals but out of all of themgoldand silver nanoparticles are important for biomedical use, a large number of ligands have been linked to nanoparticles such as peptides,sugar, proteins and DNA [7]. They have been used fordrug discovery, bioassays, active delivery of bioactive, detection, imaging and many other applications due to surface functionalization ability. Due these advantages it is an alternative to quantumdots [8]. 


\section{H.Fullerenes}

A Fullerene isentirely composed of carbon in the forms of hollow sphere, ellipsoid or tubular and many more shapes. Buckminster Fuller who designed geodesic physical structures and buildings based on this geometry, fullerenes are commonly referred to as "Buckyballs". A Buckyball is a carbon consisting hollow geometric sphere, first found in soot developed from a laboratory experiment [24]. Fullerenes are similar to carbon nanotubes in that their molecular framework is entirely composed of an extensive pconjugated carbon skeleton. They are typically synthesized by poorly understood empirical methods; for instance, the vaporization of graphite by resistive heating yields grunge from which fullerenes can be isolated chromatographically [25]. Fullerenes bind very efficiently and inactivate radicals that play a vital role in the development of diseases of the central nervous system e.g. Parkinson, Alzheimer diseases and cardiovascular diseases [26].

\section{III.APPLICATION OF PHARMACEUTICAL NANOTECHNOLOGY}

From the concept of the Nanotechnology, the current approach to Pharmaceutical therapy in which drug is systemically absorbed by whole body in order to affect a single localized organ, according to which that organ, or diseased part of it, should be targeted with molecular precision. The pharmaceuticals in current rely on slight differential selectivity of binding or uptake, and a dose sufficient to be effective against the diseased organ is likely to have significantly deleterious effects on the body as a whole when weak binding and uptake are summed over the entire rest of the body. The pharmaceutical nanotechnology has been also focusing the following applications.

\section{A.Drug discovery and design}

Nanotechnology is playing vital role for better understanding of mechanism of the drug action and identification of biomarker associated with specific disease which assist in new drug discovery and design. Byidentifying the protein present on the surface or target surface nanotechnology helps in identification and validation of target.Nanotechnology will enhance the drug discovery process, through miniaturization, automation, speed and reliability of assays. The examples of nanotechnology in drug discovery arequantum dots are used for periods ranging from milliseconds to minutes to track individual glycine receptors and to analyse their dynamics in the neuronal membrane of living cells, single walled nanotubes are successfully used to identity surface protein of pathogen. Similarly, gold nanoparticles, nanobodies which are smallest, intact, antigen-binding fragments produced by Ablynx are some commonly used nanomaterials in diagnostics [27].

\section{B.Drug Delivery}

The pharmacokinetics profile of new discovered drug entity can be modified by using Pharmaceutical nanotechnology. Nanoparticle-based drug delivery has many advantages over conventional drug delivery system, such as enhancing drugtherapeutic efficiency and pharmacological characteristics. Because nanoparticles having advantages such asmodify pharmacokinetics, improve the solubility of poorly watersoluble drugs, improve bioavailability,increase drug half-life by reducing immunogenicity, increase specificity towards the target cell or tissue therefore reducing side effects, diminish drug metabolism and enable a more controllable release of therapeutic compounds and the delivery of two or more drugs simultaneously for combination therapy [28]. The nanotechnology based drug delivery system also used for delivery of miconazole to the skin [29]. Nanotechnologies endue drug delivery system with optimized physical, chemical and biological properties can serve as effective delivery tools for currently available bioactives. Few nanotechnology based drug delivery devices are liposome,dendrimer,polymeric nanoparticles, polymer-drug conjugates, polymeric micelles, antibody- drug conjugates, which can broadly be classify as (i) stimuli sensitive delivery system, (ii)sustained and controlled delivery system, (iii) intracellular, cellular, tissue site specific targeting, (iii) multifunctional system for combined delivery of therapeutics, bio-sensing and diagnostic, and (iv)functional system for delivery of bioactives [30].

\section{C.Molecular Diagnostics}

The nano science of representing, characterizing, and quantifying subcellular biological processes in entire organisms is called as Molecular imaging. These processes include gene expression, protein-protein interaction, signal transduction, cellular metabolism, and both intracellular and intercellular trafficking. Some nanoparticles such as iron oxide nanocrystal, quantum dots and metallic nanoparticleswhich have inherent diagnostic properties. They have been successfully utilized in various magnetic resonance imagings, optical imaging, ultrasonic imaging and nuclear imaging [31]. The combination of other nanotechnology-based materialswithnanoparticles has the potential to address this emerging challenge and provide technologies that makes diagnoses possible at the level of single molecules and single cells [28]. In bio-imaging quantum dots particles such ascadmium telluride, cadmium selenide, indium phosphide, and indium arsenide serve as contrast agents, providing much higher resolution than existing fluorescent dyes [10]. 


\section{D.Tissue Engineering}

Tissue engineering makes use of artificially stimulated cell proliferation by using suitable nanomaterialbased growth factorsandscaffolds.Nanotechnology can assist to repair damaged tissueor toreproduce it. In future with more advances in tissue engineering might replace today's conventional treatments like organ transplants or artificial implants [32]. Nanotechnologies and micro technologies can be merged with biomaterials to generate scaffolds for tissue engineering that can maintain and regulate cell behaviour [33]. Nanotechnology can be used to create Nano patterns, nanofibers and control release nano particles with useful application in tissue engineering [34].

In gene therapy, using a carrier moleculea normal gene is inserted in place of an abnormal diseasecausing gene. Conventional viral vectorsuse as a carrier molecule are associated with inflammatory reactions, adverse immunologic, and diseases in the host. Nanotechnology based drug delivery systems have currently emerged as potential carrierfor effective and promising tool in systemic gene treatment. Nanoparticles composed form polymer like chitosan, poly-l-lysine and modified silica nanoparticles have been reported to have enhanced transfection efficiency and reduced cytotoxicity. Nanotechnology provides viable option as ideal vector in gene delivery [35]. Nano sized liposomes can be used for delivery of genetic material into desire cells. Liposomes incorporated with polyethylene glycol and galactose target liver cells effectively due to their rapid uptake by liver Kupffer cells. Thus gene therapy may be useful with such liposomal nanoparticles for variousliver disorders such ashereditary hemochromatosis and Wilson's disease [25]. A polymeric nanoparticle givesanti-proliferative effects by targeted delivery of gene therapy to breast cancer cells [36].

\section{E.Stem Cell Therapy}

Current research suggestsnanoparticles may be useful as effective tools for improving stem cell therapy. Chemical engineers have successfully used nanoparticles to enhance stem cells' ability to stimulate regeneration of damaged vascular tissue and reduce muscle degeneration in mice. In stem cell therapy magnetic nanoparticles coupled to antibodies are added to a blood or bone marrow sample that contains the target adult stem cells. The magnetic particles bind the target cells, which then can be recovered using a magnet. This technique is used in cell therapies to isolate adult stem cells that are then retransplanted in the patient e.g. to treat blood disorders or cardiac diseases [26]. Theuse of iron oxide nanoparticles to develop magnetodendrimers that can be used to label human neural stem cells and mesenchymal stem cells through nonspecific membrane adsorption processes [37]. The use of super paramagnetic iron oxide particles to label human mesenchymal stem cells to track their migration using MRI after transplanting it for cartilage repair [38].

\section{F.In Cancer treatment}

The research going on their conclusions shows that nanoparticles are useful for cancer treatments these includes are liposomes, quantum dots, solid lipid nanoparticles, nano shells, gold nanoparticles. Due to small size nanoparticles penetrate small capillary and are taken up by the cell which allows for efficient drug accumulation at the target sites in the body [39]. The effectiveness of nanotechnology based drug delivery systems have few advantages such as their small size, reduced drug toxicity, controlled time release of the drug and modification of drug pharmacokinetics and biological distribution overcome drawbacks of conventional anti-cancer drug delivery system [40].

\section{G.Artificial Organs and Implants}

Transplantation is often the only option for many patients those suffering from end-stage organ failure. Application of nanotechnology in artificial organ and implant development generates hope for those patients. Nanotechnology can be useful for the development of artificial organs and implants but still safety and clinical approvals yet to grant by respective regulatory authority. Current research and investigations are going on for replacement of defective or incorrectly functioning cells and organs with artificial cells [14].

\section{IV.FUTURE ASPECTS OF PHARMACEUTICAL NANOTECHNOLOGY}

Now a day's materials with nanostructure by combination with nanotechnology techniques are being used to make better composite materials, materials with enhanced catalytic activity, hardness and scratch resistance, and a wide range of consumer products that improve human life. Pharmaceutical nanotechnology has emerged as a discipline having tremendous potential as a carrier delivery of bioactives and diagnostics and provides smart materials for tissue engineering. It offers novel tools, scope and opportunities, which are anticipated to have a great impact on many areas in diagnostics of disease and treatment of diseases through its nano-engineered tools. Pharmaceutical nanotechnology gives better opportunities to enhance materials, medical devices and assist to develop novel technologies where existing and more conventional technologies may have their limits. In coming future nanotechnology will provide us the new nanotechnology such as Nano cocoons, smart medicine and nanorobots to make important contributions to disease diagnosis, detection, therapy, and prevention. 


\section{V.Conclusion}

This review summarized recent developments in oral cancer detection methods with an emphasis on nanotechnology. Nanomaterials have unique features that are attractive, and can be applied to biosensing. The development of various nanomaterials and nanotechnology has enabled detection of oral cancer biomarkers with great precision and sensitivity that could not be achieved before. The low detection limit obtained by nanotechnology is expected to contribute immensely to the early detection and accurate prognosis of oral cancers. Since it is of huge importance to be able to diagnose oral cancer as early as possible, many studies are being conducted on developing sensing mechanisms that will push down the detection limit as far down as possible. As well, various new biomarkers can be discovered and verified with such sensitive tools. It is therefore highly anticipated that in the near future, nanotechnology shall help to detect oral cancer at an early stage and monitor the disease with much greater precision. It must be however noted that these new technologies must be validated critically before applying them for clinical diagnosis. Although detecting oral cancers at early stages is very attractive, factors such as probability of getting false positive/negative and impact of nanomaterials on human and environment should be fully understood.

\section{REFERENCES}

[1] Capilla MV, Olid MNR, Gaya MVO, Botella CR, Ruiz VB. Factors related to survival from oral cancer in an Andalusian population sample. Med Oral Pathol Oral Cir Bucal 2007; 12(7):518-23.

[2] Moore SR, Johnson NW, Pierce AM, Wilson DF. The epidemiology of mouth cancer: a review of global incidence. Oral Dis 2000; 6(2):65-74

[3] Chen PH, Shieh TY, Ho PS, Tsai CC, Yang YH, Lin YC, et al. Prognostic factor associated with the survival of oral and pharyngeal carcinoma in Taiwan. BMC Cancer 2007; 7:101.

[4]González AP, López MA, Martínez LV. Comportamiento clínico y epidemiológico del cáncer de cavidad oral. Hospital Oncológico Provincial Docente María Curie. Instituto Superior de Ciencias Médicas Dr. Carlos Juan Finlay; 2005.

[5] Kingsley K, O"Malley S, Ditmyer M, Chino M. Analysis of oral cancer epidemiology in the US reveals state-specific trends: implication for oral cancer prevention. BCM Public Health 2008; 8:87.

[6] Napier SS, Speight PM. Natural history of potentially malignant oral lesions and conditions: an overview of the literature. J Oral Pathol Med 2008; 37:1-10.

[7] Cho WSC. Contribution of oncoproteomics to cancer 2. biomarker discovery. Mol Cancer 2007; $6: 25$.

[8] J. K. Elango, P. Gangadharan, S. Sumithra, and M. A. Kuriakose, "Trends of head and neck cancers in urban and rural India," Asian Pacific Journal of Cancer Prevention, vol. 7, no. 1, pp. 2006; 108112.

[9] R. Sankaranarayanan, K. Ramadas, G. Thomas et al., "Effect of screening on oral cancer mortality in Kerala, India: a clusterrandomised controlled trial," The Lancet, vol. 365, no. 9475, pp. 1927-1933, 2005.

[10] Ferlay J, Shin HR, Bray F, Forman D, Mathers C, Parkin DM. GLOBOCAN 2008 v 2.0, Cancer Incidence and Mortality
Worldwide: IARC Cancer Base No. 10 [Internet]. Lyon, France: International Agency for Research on Cancer; 2010. Available from: http://globocan.iarc.

[11] National Institutes of Health Updated October 2010.

[12] Logothetidis S. Nanotechnology in medicine: the medicine of tomorrow and nanomedicine. Hippokratia 2006; 10: 7-21.

[13] Bonoiu A, Mahajan SD, Ye L, Kumar R, Ding H, Yong K-T, et al. MMP-9 gene silencing by a quantum dot-siRNA nanoplex delivery to maintain the integrity of the blood brain barrier. Brain Research. 2009; 1282: 142-55.

[14] Shi J, Votruba AR, Farokhzad OC, Langer R. Nanotechnology in Drug Delivery and Tissue Engineering: From Discovery to Applications. Nano Letters. 2010; 10: 3223-30.

[15] Karnik R, Gu F, Basto P, Cannizzaro C, Dean L, Kyei-Manu W, et al. Microfluidic Platform for Controlled Synthesis of Polymeric Nanoparticles. Nano Letters. 2008; 8: 2906-12.

[16] Joseph K, Robert L. Responsive polymeric delivery systems. Advanced Drug Delivery Reviews. 2001;6: 19-50.

[17]Alexis F, Rhee J-W, Richie JP, Radovic-Moreno AF, Langer R, Farokhzad OC. New frontiers in nanotechnology for cancer treatment. Urologic Oncology: Seminars and Original Investigations. 2008;26: 74-85.

[18] Howarth M, Takao K, Hayashi Y, Ting A.Y. Targeting the quantum dots to the surface proteins in living cells with biotin ligases. Proc. Natl. Acad. Sci. USA 2005; 102: 7583-88.

[19]Chen, Y., Lian, G., Liao, C., Wang, W., Zeng, L., Qian, C., . . . Shuai, X. (2013). Characterization of polyethylene glycol-grafted polyethylenimine and superparamagnetic iron oxide nanoparticles (PEG-g-PEI-SPION). Journal of Gastroenterology, 48(7), 809821. doi: 10.1007/s005350120713x 10.1016/j.jconrel.2006.05.023)Google

[20] Cheng, Z., Elias, D. R., Kamat, N. P., Johnston, E. D., Poloukhtine, A., Popik, V., . . . Tsourkas, A. (2011). Improved tumor targeting of polymer-based nanovesicles using polymer-lipid blends. Bioconjug Chem, 22(10), 2021-2029. doi: $10.1021 / \mathrm{bc} 200214 \mathrm{~g}$

[21] Chetan C. Anajwala, G. K. J., S.M. Vijayendra Swamy. (2010). Current Trends of Nanotechnology for Cancer Therapy. International Journal of Pharmaceutical Sciences and Nanotechnology, 3(3), 1043-1056.

[22] Choi, Y. E., Kwak, J. W., \& Park, J. W. (2010). Nanotechnology for early cancer detection. Sensors (Basel), 10(1), 428-455. doi: $10.3390 / \mathrm{s} 100100428$

[23] Cridge, B. J., Larsen, L., \& Rosengren, R. J. (2013). Curcumin and its derivatives in breast cancer: Current developments and potential for the treatment of drug-resistant cancers. Oncology Discovery, 1(1), 6. doi: 10.7243/2052-6199-1-6.

[24] Yan, J., Zheng, X., Liu, Z., Yu, J., Deng, Z., Xue, F., . . . Li, G. (2016). A multicenter study of using carbon nanoparticles to show sentinel lymph nodes in early gastric cancer. Surg Endosc, 30(4), 1294-1300. doi: 10.1007/s00464-015-4358-8

[25] Ye, M. X., Li, Y., Yin, H., \& Zhang, J. (2012). Curcumin: updated molecular mechanisms and intervention targets in human lung cancer. Int J Mol Sci, 13(3), 3959-3978. doi: 10.3390/ijms 13033959

[26] Ying Wu, W. W., Yinting Chen, Kaihong Huang, Xintao Shuai, Qikui Chen, Xuexian Li, Guoda Lian. (2010). The investigation of polymer-siRNA nanoparticle for gene therapy of gastric cancer in vitro. Int J Nanomedicine, 5, 129-136.

[27] Yun-Peng Zhang, P. S., Xu-Rui Zhang, Wu-Li Yang and ChengShuai Si. (2013). Synthesis of CdTe quantum dot-conjugated CC49 and their application for in vitro imaging of gastric adenocarcinoma cells. Nanoscale Res Lett, 8 .

[28] Zhang, W., Zhang, Z., \& Zhang, Y. (2011). The application of carbon nanotubes in target drug delivery systems for cancer 
therapies. Nanoscale Res Lett, 6, 555. doi: 10.1186/1556-276X6555

[29] Zhang, Z., Niu, B., Chen, J., He, X., Bao, X., Zhu, J., . . Li, Y. (2014). The use of lipid-coated nanodiamond to improve bioavailability and efficacy of sorafenib in resisting metastasis of gastric cancer. Biomaterials, 35(15), 4565-4572. doi: 10.1016/j.biomaterials.2014.02.024.

[30] Thakor, A. S., \& Gambhir, S. S. (2013). Nanooncology: the future of cancer diagnosis and therapy. CA Cancer J Clin, 63(6), 395418. doi: 10.3322/caac.21199

[31] Thomas, D. G., Pappu, R. V., \& Baker, N. A. (2011). NanoParticle Ontology for cancer nanotechnology research. J Biomed Inform, 44(1), 59-74. doi: 10.1016/j.jbi.2010.03.001

[32] Tokuhara, T., Tanigawa, N., Matsuki, M., Nomura, E., Mabuchi, H., Lee, S. W., . . . Narabayashi, I. (2008). Evaluation of lymph node metastases in gastric cancer using magnetic resonance imaging with ultrasmall superparamagnetic iron oxide (USPIO): diagnostic performance in post-contrast images using new diagnostic criteria. Gastric Cancer, 11(4), 194-200. doi: 10.1007/s10120-008-0480-9

[33] Trickler, W. J., Khurana, J., Nagvekar, A. A., \& Dash, A. K. (2010). Chitosan and glyceryl monooleate nanostructures containing gemcitabine: potential delivery system for pancreatic cancer treatment. AAPS PharmSciTech, 11(1), 392-401. doi: 10.1208/s12249- 010-9393-0

[34] Veiseh, O., Kievit, F. M., Ellenbogen, R. G., \& Zhang, M. (2011). Cancer cell invasion: treatment and monitoring opportunities in nanomedicine. Adv Drug Deliv Rev, 63(8), 582-596. doi: 10.1016/j.addr.2011.01.010

[35] Vergaro, V., Scarlino, F., Bellomo, C., Rinaldi, R., Vergara, D., Maffia, M., . . . Leporatti, S. (2011). Drug-loaded polyelectrolyte microcapsules for sustained targeting of cancer cells. Adv Drug Deliv Rev, 63(9), 847-864. doi: 10.1016/j.addr.2011.05.007

[36] Vladimir N. Anisimov, I. A. V., Andrei V. Panchenko1, Irina G. Popovich and, \& Zabezhinski, M. A. (2012). Light-at-NightInduced Circadian Disruption, Cancer and Aging Current Aging Science, 5, 170-177.

[37] Wang, J. J., Zeng, Z. W., Xiao, R. Z., Xie, T., Zhou, G. L., Zhan, X. R., \& Wang, S. L. (2011). Recent advances of chitosan nanoparticles as drug carriers. Int J Nanomedicine, 6, 765-774. doi: 10.2147/IJN.S17296. 